\title{
Primary Malignant Melanoma Limited to the External Auditory Canal: A Rare Presentation
}

\author{
Dillip Kumar Samal ${ }^{1}$ C. Preetam ${ }^{1}$ Anjan Kumar Sahoo ${ }^{2}$ \\ ${ }^{1}$ Department of ENT \& Head and Neck Surgery, AIIMS, \\ Bhubaneswar, Orissa, India \\ 2Department of ENT \& Head and Neck Surgery, Maulana Azad \\ Address for correspondence Dillip Kumar Samal, MS, Department of \\ ENT \& Head and Neck Surgery, Room no. 413, Academic Building, AlIMS, \\ Bhubaneswar 751019, Orissa, India (e-mail: drdillipsamal@gmail.com).
}

Medical College, New Delhi, India

Ann Otol Neurotol ISO 2021;4:33-35.

\begin{abstract}
Keywords

- superficial parotidectomy

- lateral temporal bone resection

- neck dissection

- external auditory canal

Malignant melanoma limited to the external auditory canal is very rare. These patients present relatively late as compared with melanoma involving other subsites of external ear. However, the tumor is slow-growing but shows aggressive behavior with a poor prognosis when presented late. We have a 44 -year-old female who presented with a blackish lesion, which was bleeding on and off from her left ear. She was managed with wide local excision, preserving maximum part of external auditory canal cartilage. The histopathology of the lesion was suggestive of malignant melanoma. After a thorough evaluation, she was kept under close follow-up. Malignant melanoma of external auditory canal shows poor prognosis usually, mainly because of late presentation. Thus, early diagnosis is crucial, as in our case, where wide local excision was sufficing, and the patient is disease-free after 4 years of follow-up.
\end{abstract}

\section{Introduction}

Malignant melanoma is an uncommon malignancy in the head and neck region. Among the various subsites, external auditory canal melanoma is an infrequent occurrence. Maximum 30 cases have been reported in the literature to date. Compared with other subsites, melanoma of external auditory canal presents late and has a poor prognosis despite extensive surgery and adjuvant therapy. ${ }^{1}$

It may present as a bleeding polyp, mass in the external auditory canal, ulceration, pain, discharge, or hearing loss in the affected ear. Sometimes, it can metastasize locoregionally or present with distant metastasis at the time of diagnosis. It is treated with lateral temporal bone resection along with superficial parotidectomy and neck dissection in advanced cases. Adjuvant radiotherapy, chemotherapy, and immunotherapy may be added if required. It is an aggressive malignancy with a poor prognosis.

We had a middle-aged female patient present to us with a bleeding polypoidal mass in her left ear, which was managed surgically.

\section{Case Report}

A 44-year-old female presented to our outpatient department with ear discomfort and occasional bleeding from her left ear for 10 to 12 months. She had a feeling of decreased hearing in the same ear for 3 to 4 months before presentation. Local examination revealed a blackish mass in her left external auditory canal ( $\mathbf{- F i g . 1}$ ) that was bleeding on manipulation. The tuning fork test and audiometry showed mild conductive hearing loss in the ipsilateral ear, and the right ear hearing
Published online September 29, 2021
DOI https://doi.org/ 10.1055/s-0041-1735991 ISSN 2581-9607 (c) 2021. Indian Society of Otology.

This is an open access article published by Thieme under the terms of the Creative Commons Attribution-NonDerivative-NonCommercial-License, permitting copying and reproduction so long as the original work is given appropriate credit. Contents may not be used for commercial purposes, or adapted, remixed, transformed or built upon. (https://creativecommons.org/licenses/by-nc-nd/4.0/).

Thieme Medical and Scientific Publishers Pvt. Ltd. A-12, 2nd Floor, Sector 2, Noida-201301 UP, India 
level was normal. High-resolution and contrast-enhanced computer tomography of the temporal bone was done. The scan showed an enhancing mass in the right external auditory canal at its opening. The bony external auditory canal, middle ear, mastoid, and surrounding structures, including parotid glands, were normal. No cervical lymphadenopathy was identified on palpation. Laboratory investigations and other systemic examinations showed no significant abnormality.

After taking informed and written consent, wide excision of the black bleeding mass $(23 \times 6 \times 7 \mathrm{~mm})$ was done under local anesthesia (-Fig. 2). It was arising from the floor of the external auditory canal with narrow attachment. The cartilage base of the lesion was excised and cauterized. Surrounding cartilage from the external auditory canal and concha was excised, preserving the tragus. The ear canal was packed with an antibiotic-soaked pack, which was removed after 10 days. The histopathology of the excised specimen showed invasion of the basal layer of epidermis at places with tumor cells. The tumor cells are strongly and diffusely positive for HMB-45 and S-100 (-Fig. 3) and negative for synaptophysin and chromogranin. The perichondrium and underlying excised cartilage were free. The overall histopathological features were consistent with malignant melanoma.

After histopathological diagnosis, the patient was evaluated immediately with contrast-enhanced computer tomography

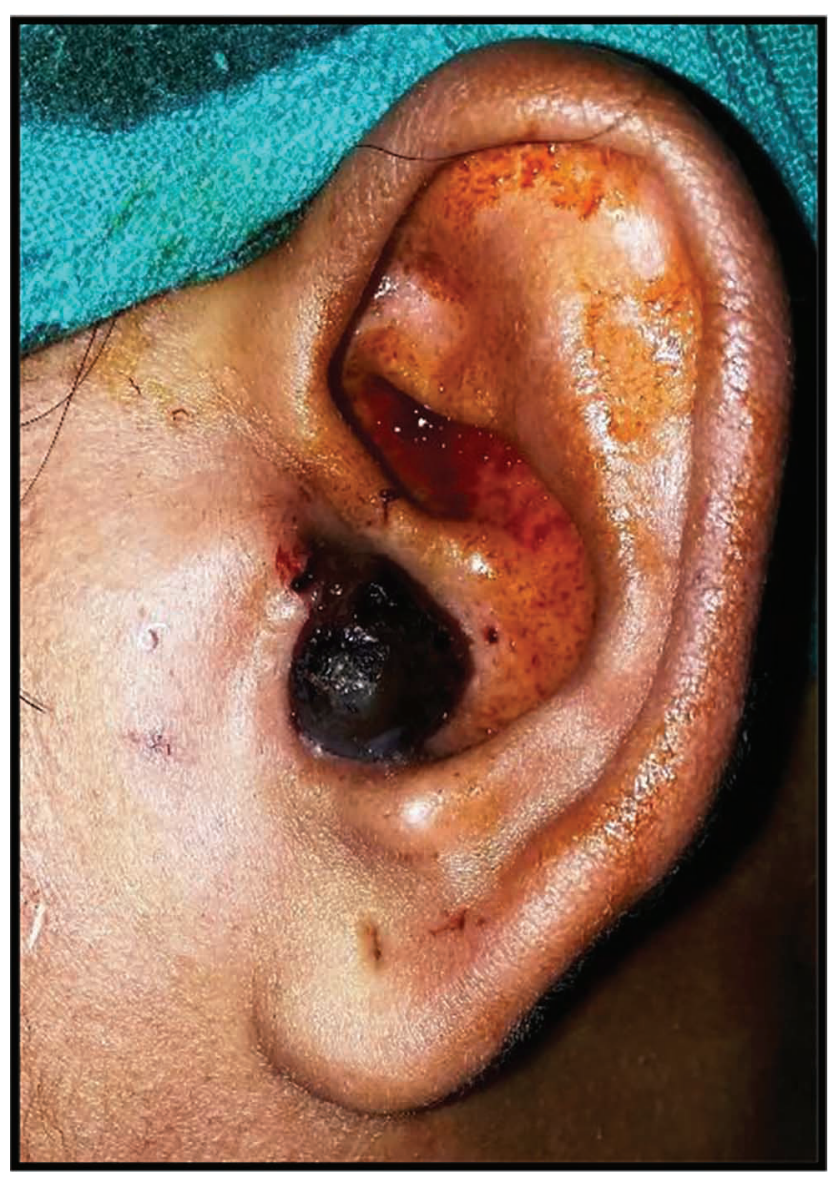

Fig. 1 Malignant melanoma occluding left external auditory canal.

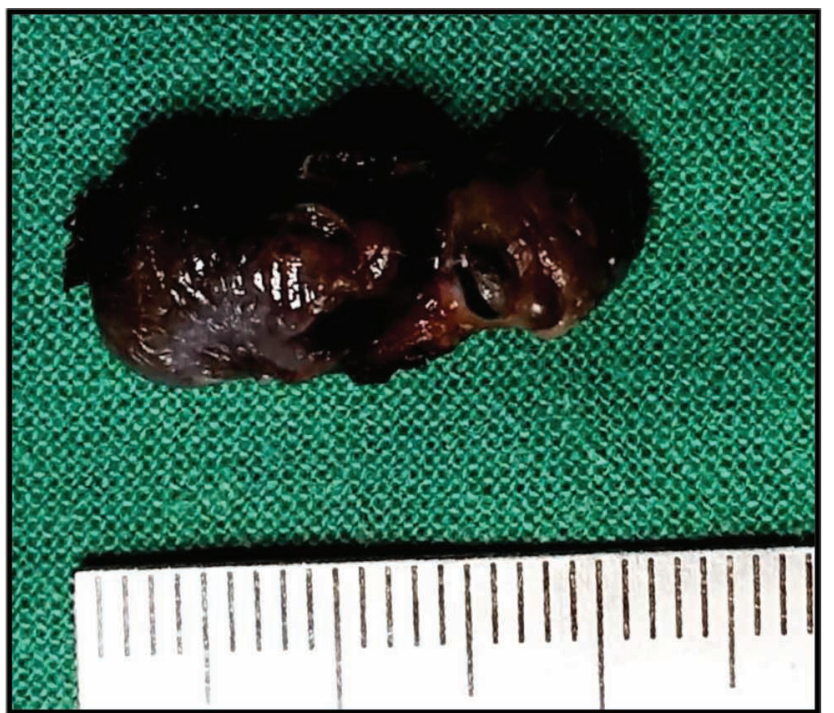

Fig. 2 Excised specimen from left external auditory canal $(2.3 \times$ $0.6 \times 0.7 \mathrm{~cm})$.

of the abdomen and chest to rule out any distant organ metastasis. Positron emission tomography scan was done after 6 weeks and no residual local lesion or distant metastasis was identified. The oncology board's opinion was taken. The patient was kept under close observation with monthly periodic evaluation in the initial 1 year and then for 3 to 6 monthly follow-up. At 1 year, she was evaluated with contrast-enhanced computer tomography of the abdomen and ultrasound of the neck and chest X-ray. The patient did not show any locoregional recurrence or any features of distant metastasis till the last follow-up, that is, 4 years after her illness.

\section{Discussion}

Malignant melanoma is a rare and aggressive tumor arising from melanocytes. Malignant melanoma of the ear constitutes 7 to $14 \%$ of all head and neck region melanoma. Ear comprises 1 to $4 \%$ of all skin melanomas, of which helix constitutes the commonest site followed by ear lobule. ${ }^{2}$ The external auditory canal is a very rare site of tumor occurrence compared with other subsites of the external ear. Among various histological types, superficial spreading type is the commonest variant found, and the median survival time is 7.9 years. ${ }^{2,3}$

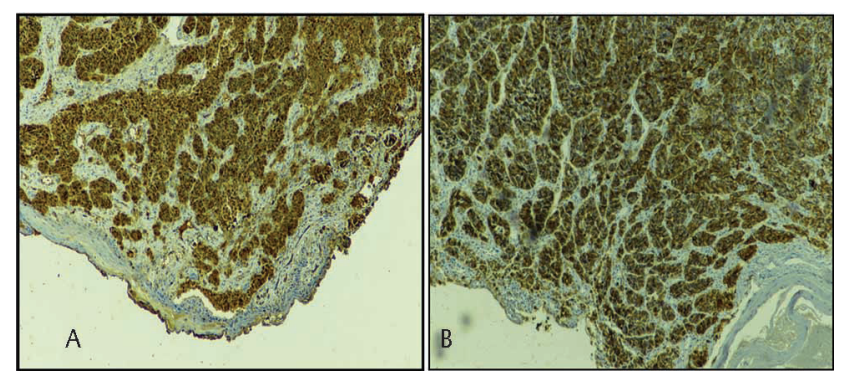

Fig. 3 (A) Tumor cells showing strongly immunopositivity for S-100 protein. (B) Tumour cells showing strong and diffuse immunopositivity for HMB 45. 
Malignant melanoma may present with a bleeding polyp in the ear or as an ulcerated mass, pre- and postaural swelling, and pain. It may erode the ear canal and may invade the parotid gland in the advanced stage. Regional cervical lymph node metastasis is uncommon.

Early lesions can be managed by cartilage sparing wide local excision of the tumor followed by reconstruction if required with a good survival rate. ${ }^{4}$ Neck node dissection is not recommended in node-negative patients. ${ }^{3}$ Local recurrence and distant metastasis are not uncommon and have a very poor prognosis. In patients with extensive disease, the prescribed treatment modality includes wide local excision with lateral temporal bone resection, superficial parotidectomy, selective/functional neck dissection with postoperative radiation, and chemotherapy. But these patients have an abysmal prognosis despite the multimodality approach. Tumor thickness and Clark level of invasion are the important risk factors for disease-specific survival. ${ }^{5}$

In our case, the tumor was limited to the cartilaginous part of the external auditory canal. Underlying perichondrium, cartilage, and the bony canal were spared, and there was no regional cervical nodal metastasis. The perichondrium acts as a barrier for the spread in early cases. Thus, wide local excision preserving the cartilage can be considered sufficient in early melanoma cases involving the helix. ${ }^{4}$ Therefore, wide local excision with close observation was oncologically adequate. She is disease-free after 4 years of her illness.

\section{Conclusion}

Ear melanoma involving the external auditory canal is very rare. The prognosis is poor in patients with an extensive disease with distant metastasis at presentation despite extensive surgery and multimodality treatment. When presented early, wide local excision with a negative margin is sufficient. Neck dissection is usually not needed in patients without metastatic cervical lymphadenopathy. Thus, early diagnosis is the key for better survival, and extensive surgery is not usually required in every case, as was the initial protocol.

\section{Conflict of Interest}

None.

\section{References}

1 Appelbaum EN, Gross ND, Diab A, Bishop AJ, Nader ME, Gidley PW. Melanoma of the external auditory canal: a review of seven cases at a tertiary care referral center. Laryngoscope 2021;131(1):165-172

2 Toia F, Garbo G, Tripoli M, Rinaldi G, Moschella F, Cordova A. A systematic review on external ear melanoma. J Plast Reconstr Aesthet Surg 2015;68(7):883-894

3 Ravin AG, Pickett N, Johnson JL, Fisher SR, Levin LS, Seigler HF. Melanoma of the ear: treatment and survival probabilities based on 199 patients. Ann Plast Surg 2006;57(1):70-76

4 McCarty MA, Lentsch EJ, CerratiEW,Stadelmann WK. Melanoma of the ear: results of a cartilage-sparing approach to resection. Eur Arch Otorhinolaryngol 2013;270(11):2963-2967

5 Jahn V, Breuninger H, Garbe C, Moehrle M. Melanoma of the ear: prognostic factors and surgical strategies. $\mathrm{Br}$ J Dermatol 2006;154(2):310-318 